\title{
New Imaging modality for surface and sub-surface imaging using Scanning Transmission Helium Ion Microscopy
}

Saba Tabean ${ }^{1}$, Santhana Eswara ${ }^{2}$, Michael Mousley ${ }^{2}$, Olivier De Castro ${ }^{3}$, Jean-Nicolas Audinot ${ }^{3}$ and Tom Wirtz ${ }^{1}$

${ }^{1}$ Luxembourg Institute of Science and Technology (LIST), Belvaux, Luxembourg, ${ }^{2}$ Luxembourg Institute of Science and Technology, Belvaux, Luxembourg, ${ }^{3}$ Luxembourg Institute of Science and Technology (LIST), Luxembourg

Helium Ion Microscopy (HIM) has been receiving increasing scientific interest because of its highresolution imaging and nanofabrication capabilities. The microscope comprises a Gas Field Ion Source (GFIS). The high electric field at the apex of the GFIS ionizes helium atoms and the $\mathrm{He}^{+}$ions are rasterscanned over the sample to generate secondary electrons (SE), which are collected to obtain conventional SE images in HIM [1]. Various detectors have also been developed to detect other type of signals in a HIM such as backscattered particles [2], ionoluminescence [3], transmitted ions [4], and mass filtered secondary ions [5]. This paper presents novel complementary approaches to characterize materials using the GFIS based platform integrated with Secondary Ion Mass Spectrometry (SIMS) and Scanning Transmission HIM (STHIM) namely the npSCOPE developed by Luxembourg Institute of Science and Technology (LIST).

The ability to image sub-surface features such as buried crystalline defects or precipitates in a HIM is necessary for targeted local nanoscale structural and chemical analysis and in applications such as circuitediting. Conventional SE imaging mode in a HIM provides information only about the surface. STHIM imaging mode addresses this important characterization challenge. Other groups have previously reported the use of HIM in transmission mode, however, direct quantitative detection of transmitted ions for both bright-field and dark-field imaging was lacking until recently [6]. By using a delay-line detector, the possibility to obtain quantitative images using transmitted helium ions in the keV energy range is now demonstrated on the npSCOPE instrument. By obtaining 2D images of the detector at each single position of the raster, a 4D dataset is obtained. With this, it is now possible to probe the transmitted ion intensity and the scattering angle at nanoscale and at individual pixel level. This unprecedented level of quantitative information opens the door to advanced experiments in both fundamental physics as well as in materials science.

In this paper, we extend STHIM imaging in npSCOPE for quantitative analysis of ion channelling through crystalline defects (e.g. twin boundary) using polycrystalline Au and nanocrystalline Si as model samples. In Fig. 1 and Fig. 2, standard SE images are compared with the transmitted ions images of the same area. The images are taken with $25 \mathrm{keV} \mathrm{He}^{+}$with 60fA beam current and for the STHIM images pixel dwell time of $110 \mu$ s used with a single scan. As it can be seen in both Figures, the STHIM images reveal hidden structures and show new contrasts (mainly from channelled transmitted ions) from the sample in comparison to the SE images. In addition, new results obtained using SIMS focal plane detector will also be presented to summarize the new imaging and analysis modalities now possible in the HIM and npSCOPE instruments. 


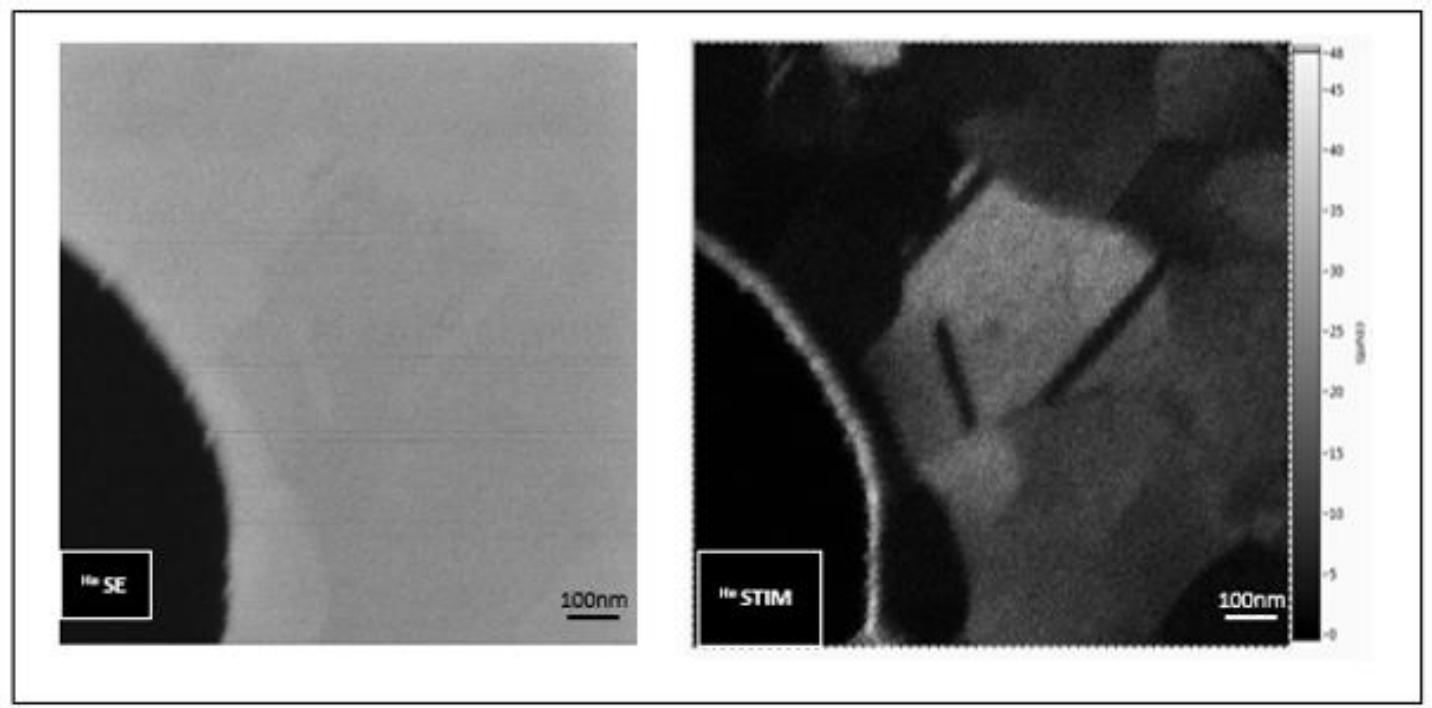

Figure 1. Comparison of a SE image with STHIM image for a polycrystalline gold with a thickness $~ 50$ nm.
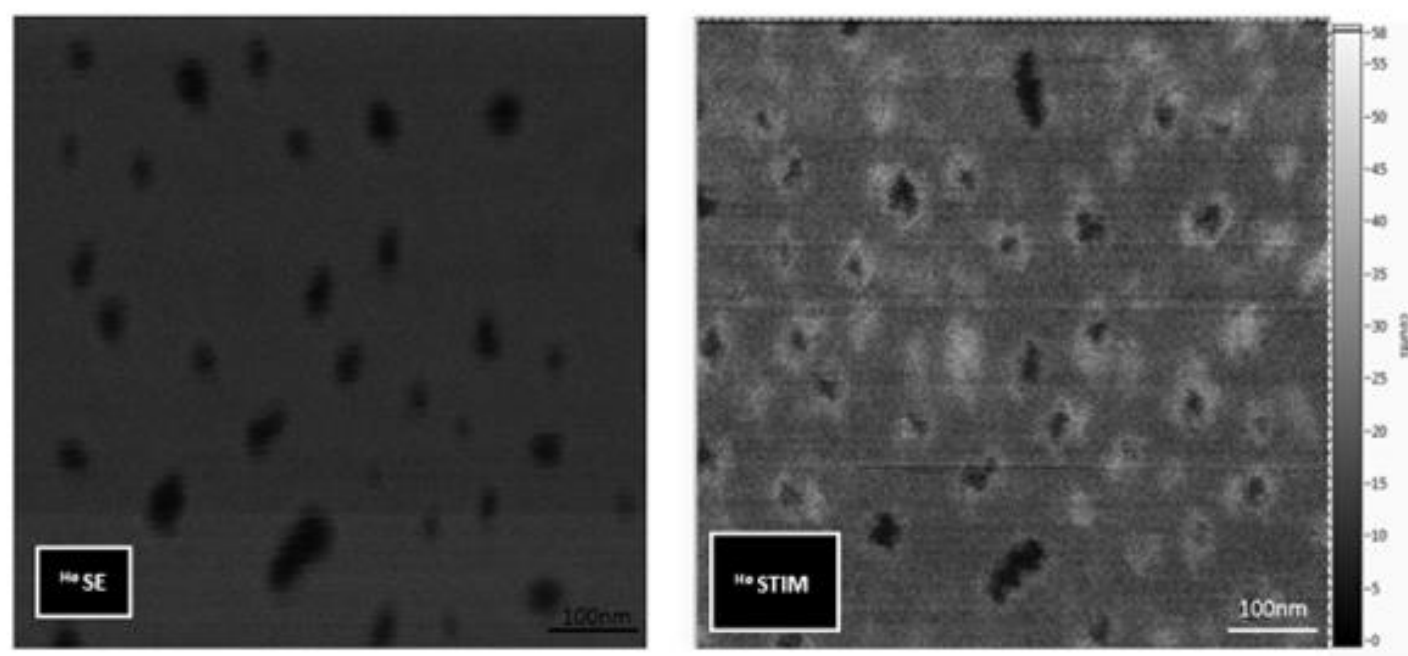

Figure 2. Comparison of a SE image with STHIM image for a nanocrystalline porous silicon membrane with a thickness $~ 30 \mathrm{~nm}$.

\section{References}

[1] Hlawacek, G., Veligura, V., van Gastel, R., \& Poelsema, B. (2014). Helium ion microscopy. Journal of Vacuum Science \& Technology B, Nanotechnology and Microelectronics: Materials, Processing, Measurement, and Phenomena, $32(2), 020801$.

[2] van Gastel, R., Hlawacek, G., Dutta, S., \& Poelsema, B. (2015). Backscattered helium spectroscopy in the helium ion microscope: Principles, resolution and applications. Nuclear Instruments and Methods in Physics Research Section B: Beam Interactions with Materials and Atoms, 344, 44-49.

[3] Veligura, V., Hlawacek, G., van Gastel, R., Zandvliet, H. J., \& Poelsema, B. (2014). A high resolution ionoluminescence study of defect creation and interaction. Journal of physics: Condensed matter, 26(16), 165401. 
[4] Serralta, E., Klingner, N., De Castro, O., Mousley, M., Eswara, S., Pinto, S. D., ... \& Hlawacek, G. (2020). Scanning transmission imaging in the helium ion microscope using a microchannel plate with a delay line detector. Beilstein Journal of Nanotechnology, 11(1), 1854-1864.

[5] Wirtz, T., De Castro, O., Audinot, J. N., \& Philipp, P. (2019). Imaging and analytics on the helium ion microscope. Annual Review of Analytical Chemistry, 12, 523-543.

[6] Serralta, E., Klingner, N., De Castro, O., Mousley, M., Eswara, S., Pinto, S. D., ... \& Hlawacek, G. (2020). Scanning transmission imaging in the helium ion microscope using a microchannel plate with a delay line detector. Beilstein Journal of Nanotechnology, 11(1), 1854-1864.

[7] This work was funded by the Luxembourg National Research Fund (FNR) by the grant PRIDE17/12246511/PACE 